\title{
Electrical Characterization of Metastable Defects Introduced in GaN by Eu-Ion Implantation
}

\author{
F. Danie Auret ${ }^{1, a}$, Walter E. Meyer ${ }^{1, b+}$, M. Diale ${ }^{1, c}$, P. J. Janse van Rensburg ${ }^{1, d}$, \\ S.F. Song ${ }^{2, e}$, K. Temst ${ }^{2, f}$ and A. Vantomme ${ }^{2, g}$ \\ ${ }^{1}$ Physics Department, University of Pretoria, Pretoria, South Africa \\ ${ }^{2}$ Instituut voor Kern- en Stralingsfysica, K.U.Leuven, Celestijnenlaan 200 D, 3001 Leuven, \\ Belgium
}

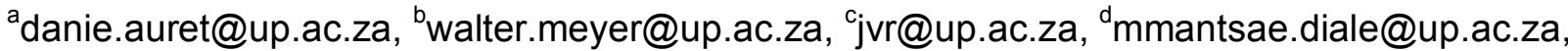

esfsong@bjtu.edu.cn, ${ }^{9}$ kristiaan.temst@fys.kuleuven.be, ${ }^{9}$ andre.vantomme@fys.kuleuven.be

Keywords: Ion implantation, GaN, metastable defects, DLTS.

\begin{abstract}
Gallium nitride (GaN), grown by HVPE, was implanted with $300 \mathrm{keV}$ Eu ions and then annealed at $1000{ }^{\circ} \mathrm{C}$. Deep level transient spectroscopy (DLTS) and Laplace DLTS (L-DLTS) were used to characterise the ion implantation induced defects in GaN. Two of the implantation induced defects, E1 and E2, with DLTS peaks in the $100-200 \mathrm{~K}$ temperature range, had DLTS signals that could be studied with L-DLTS. We show that these two defects, with energy levels of $0.18 \mathrm{eV}$ and $0.27 \mathrm{eV}$ below the conduction band, respectively, are two configurations of a metastable defect. These two defect states can be reproducibly removed and re-introduced by changing the pulse, bias and temperature conditions, and the transformation processes follow first order kinetics.
\end{abstract}

\section{Introduction}

$\mathrm{GaN}$ is a material that has received a lot of attention for its potential use in optoelectronic applications such as light emitting diodes, detectors and laser diodes [1]. As a consequence of its wide $(3.4 \mathrm{eV})$ direct band-gap, GaN demonstrates low thermal carrier generation rates and lower luminescence thermal quenching when compared to $\mathrm{Si}$, for example. This makes $\mathrm{GaN}$ a suitable host for optical dopants introduced by ion implantation. Rare-earth (RE) elements have already been used in optoelectronic devices, such as the Nd:YAG laser, YOBr:Eu phosphors or Er in optical fibers [2]. These elements have partially filled inner $4 \mathrm{f}$ electron shells shielded by completely filled outer $5 \mathrm{~s}$ and $5 \mathrm{p}$ shells. Intra $4 \mathrm{f}$ electronic transitions result in very sharp optical emission at wavelengths ranging from ultraviolet to infrared covering the visible spectrum. The wavelength of the emitted light is almost independent of the host material. However, the host determines radiative transition probabilities and thereby plays an important role on the luminescence output. Filhol et al. [3] have calculated that $\mathrm{Eu}, \mathrm{Er}$ and $\mathrm{Tm}$ will introduce donor levels at $0.2 \mathrm{eV}$ below the conduction band. Up to now, little experimental work has been performed to show the existence of such a donor level. Mamor et al. has suggested that a level at $0.36 \mathrm{eV}$ below the conduction band of Eu- implanted GaN may be Eu-related [4]. Song et al. reported that implantation of Er and Pr induce donor levels at 0.188 and $0.190 \mathrm{eV}$, respectively, below the conduction band of $\mathrm{GaN}$ [5]. However, Janse van Rensburg et al. [6] observed similar levels after Xe implantation, leading to the conclusion that the reported levels are due to implantation damage.

In this work we show that $300 \mathrm{keV} \mathrm{Eu}$ implantation, followed by $1000{ }^{\circ} \mathrm{C}$ annealing introduces, among others, a metastable defect with two configurations that have levels at $0.18 \mathrm{eV}$ and $0.27 \mathrm{eV}$ below the conduction band. The reversible removal and introduction of these defect levels is illustrated and discussed. 


\section{Experimental procedure}

For this experiment we have used HVPE grown GaN with a free carrier density of $4 \times 10^{15} \mathrm{~cm}^{-3}$. The GaN was implanted with $300 \mathrm{keV}$ Eu ions (with an ion beam incidence $10^{\circ}$ away from the $c$ axis) at a fluence of $5 \times 10^{13} \mathrm{~cm}^{-2}$ and then annealed at $1000{ }^{\circ} \mathrm{C}$ for $60 \mathrm{~min}$ in a flow of nitrogen. Thereafter, the implanted as well as control (unimplanted) samples were chemically cleaned and Ohmic contacts were fabricated on them by evaporating Ti/Al/Ni/Au $(15 \mathrm{~nm} / 220 \mathrm{~nm} / 40 \mathrm{~nm} / 50 \mathrm{~nm})$ in an electron-beam deposition system and then annealing them in $\mathrm{Ar}$ at $500{ }^{\circ} \mathrm{C}$ for 5 minutes. Finally, circular Ni/Au (30 nm/100 nm) Schottky barrier diodes (SBDs), $0.6 \mathrm{~mm}$ in diameter, were fabricated on the $\mathrm{GaN}$ samples by resistive evaporation. Current-voltage (I-V) measurements indicated that the quality of the SBD were high with leakage currents of below $10^{-8} \mathrm{~A}$ at $1 \mathrm{~V}$ reverse bias (at room temperature). Conventional deep level transient spectroscopy (DLTS), high-resolution Laplace DLTS (L-DLTS) [7], as well as capacitance vs temperature (C-T) measurements, were used the characterize the defects in the as-grown and ion-implanted GaN.

\section{Results}

Fig. 1 shows the DLTS spectra of as grown and Eu-implanted GaN. The three most characteristic peaks of defects in as-grown HVPE GaN were present above $300 \mathrm{~K}$ and are not shown here since we shall focus on the shallower implantation induced defects. Note that the control sample contained a shallow level with a DLTS peak at $150 \mathrm{~K}$ (Fig. 1, curve (a)). The two most prominent defect peaks observed in implanted and annealed GaN were E1 and E2, with activation enthalpies of 0.18 $\mathrm{eV}$ and $0.27 \mathrm{eV}$, respectively, as determined from standard Arrhenius plots. The signature of the E1 was very similar to that of the $0.19 \mathrm{eV}$ defect reported before $[5,6]$ but it was argued that it is not Eu-related, instead damage related because it was also present in Xe-implanted GaN [6].

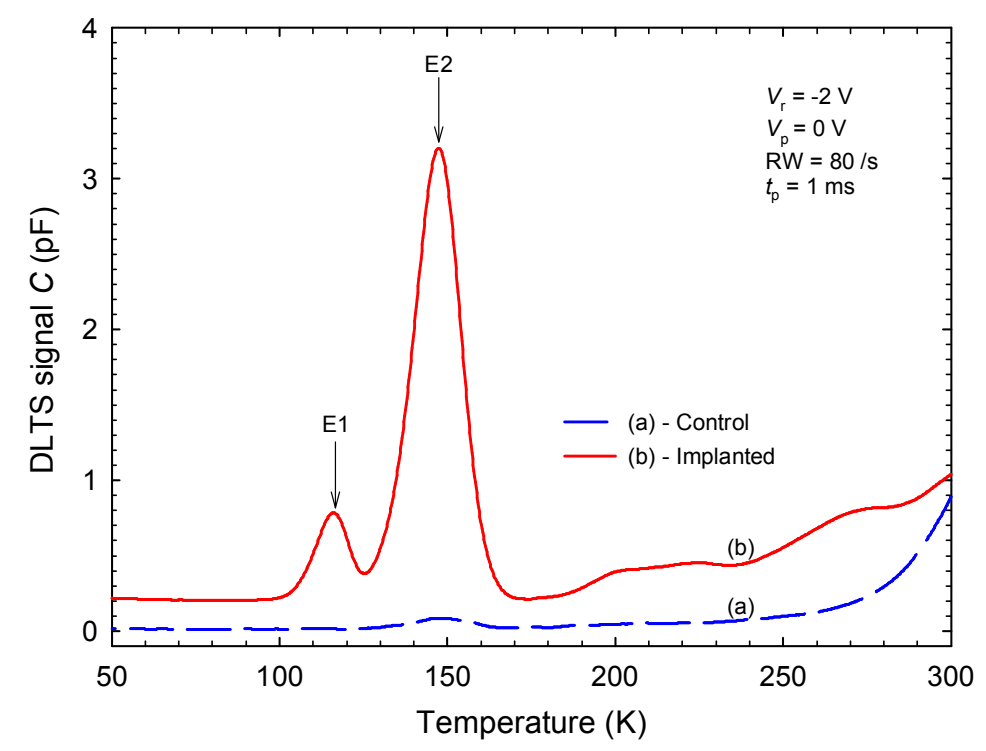

Fig. 1. DLTS spectra of control and annealed, implanted GaN SBDs, showing the two most prominent defects, E1 and $\mathrm{E} 2$, in the implanted GaN. The control sample contained a small defect with a peak at about the same temperature as that of the E2. Here, a peak height of $1 \mathrm{pF}$ coresponds to a defect density of about $8 \times 10^{14} \mathrm{~cm}^{-3}$.

These two defect levels exhibit a very interesting metastable behaviour. Fig. 2 (a) shows L-DLTS spectra recorded using different filling pulse widths at $135 \mathrm{~K}$. Two observations can be made from this Figure. Firstly, whereas E1 had a single DLTS peak associated with it, E2 consisted of two to three peaks. Secondly, the peak position of E1 is fixed for all pulse widths, whereas the position of the E2 group of peaks collectively moves to lower emission rates for longer filling pulse widths. This is reminiscent of negative $U$ behaviour where the capture of additional charge leads to a level further away from the conduction band [8]. In Fig. 2 (b) we plot the peak heights of E1 and E2 as function of the filling pulse width at $120 \mathrm{~K}$. It is clear that for very long pulses $(>1000 \mathrm{~ms})$ only E2 was observed. Note that at $135 \mathrm{~K}$ only the E2 was observed already for a $20 \mathrm{~ms}$ pulse, pointing to the temperature dependence of the process. As the pulse width was reduced, the peak height of E2 
was reduced and that of E1 increased. For pulses shorter than $0.05 \mathrm{~ms}$ E1 was maximized, E2 was minimized and the E1 amplitude was the same as that of E2 after very long filling pulses. The remaining fraction of E2 after application of short pulses is most probably not part of E2, but rather the small defect present in the unimplanted (control) sample with a small peak at almost the same temperature as E2 (see Fig. 1). It is interesting to note that the sum of the amplitudes of E1 and E2 remained constant. The results in Figs 2 (a) and (b) suggest that E1 and E2 are the energy levels associated with two different configurations of the same metastable defect.

(a)

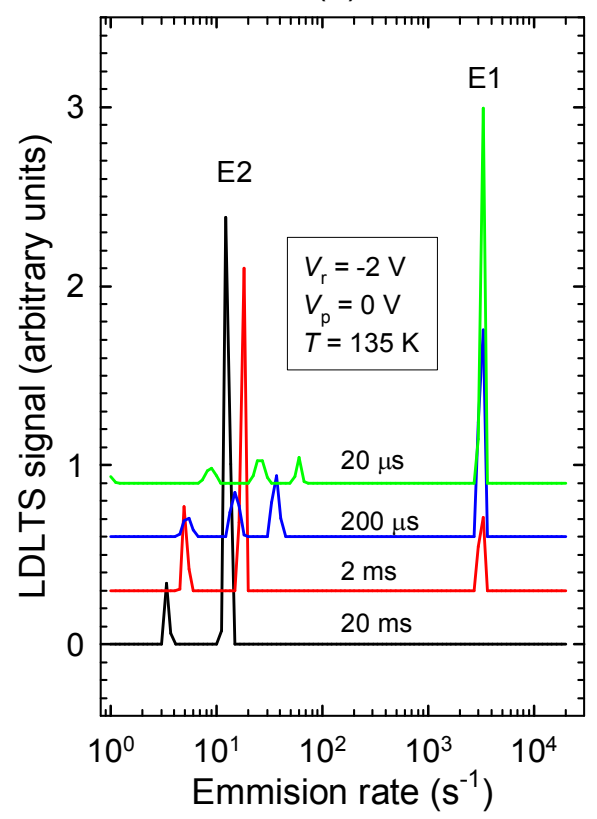

(b)

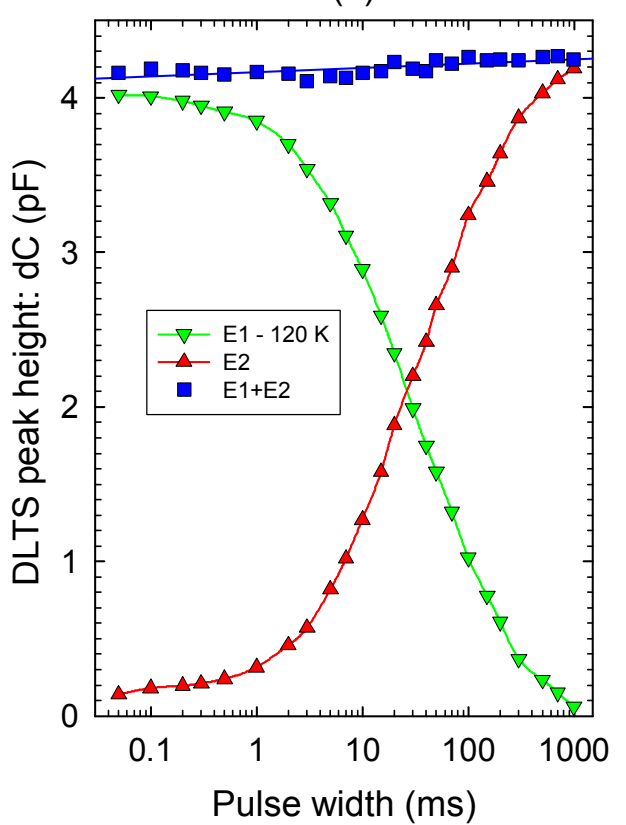

Fig. 2. (a):

L-DLTS spectra of E1 and E2 for different filling pulse widths, as indicated in the figure, record-ed at $135 \mathrm{~K}$;

Fig. 2 (b):

DLTS peak heights of E1 and E2 as function of filling pulse width, record-ed at $120 \mathrm{~K}$. The sum, E1 + E2, is also plotted.

Metastable defects are conventionally studied using zero-bias (ZB) and reverse bias (RB) temperature annealing cycles. We have performed a systematic annealing study for this defect pair. Capacitance - temperature (C-T) scans under different bias conditions were used to demonstrate the metastable behaviour of the E1 and E2 defect pair.

The first cycle was to transform all E2 to E1 and entailed annealing of the sample at $150 \mathrm{~K}$ or above under RB, i.e. under $-2 \mathrm{~V}$ bias. Next, the sample was cooled down under RB to $50 \mathrm{~K}$. At 50 $\mathrm{K}$ a zero bias $(\mathrm{ZB})$ pulse $(1 \mathrm{~s})$ was applied to the sample to fill all possible traps that emit electrons at higher temperatures. Finally, a C-T scan up in temperature under RB to $150 \mathrm{~K}$ was recorded: curve (a) in Fig. 3. The sharp increase in capacitance between $80 \mathrm{~K}$ and $90 \mathrm{~K}$ is due to the E1 emitting electrons captured during the filling pulse at $50 \mathrm{~K}$. Note that the capacitance in both cases recovered to approximately the same level at $130 \mathrm{~K}$ and there is no sign of a capacitance change at higher temperatures that would correspond to emission from E2. From this we conclude that annealing at $150 \mathrm{~K}$ under RB transformed E2 into E1. Next, the E2 was gradually re-introduced by ZB annealing in the temperature range $86 \mathrm{~K}-96 \mathrm{~K}$. First, all of E2 was again removed by annealing under RB at $150 \mathrm{~K}$. Then the sample was cooled under RB to the temperature where the transformation was to be performed, e.g. $86 \mathrm{~K}$. Once the temperature had stabilized, ZB was applied for a given time, $t$ (typically $10 \mathrm{~ms}-20000 \mathrm{~s}$ ). Following this, the RB was re-applied and the sample was rapidly cooled to $50 \mathrm{~K}$. Here, a filling pulse of $1 \mathrm{~s}$ was applied and then a C-T scan was recorded upwards in temperature under RB. This cycle was repeated for different $\mathrm{ZB}$ annealing times, $t$, at temperature $T=86 \mathrm{~K}$. The results of this are the curves (a) - (d) in Fig. 3. It is clear that for a long enough ZB anneal, the E1 had completely transformed to E2.

The inverse transformation process, namely first transforming all of E1 to E2 under ZB and then re-introducing $\mathrm{E} 1$ by annealing under $\mathrm{RB}$, was found to be conveniently measurable between $96 \mathrm{~K}$ and $106 \mathrm{~K}$. All of the E1 could be transformed to E2 by annealing under ZB above $160 \mathrm{~K}$. E1 was 
then re-introduced by annealing at $\mathrm{RB}$ between temperatures of $98 \mathrm{~K}$ and $106 \mathrm{~K}$ for different times. We have found that both these processes follow first order kinetics with activation energies of $0.18 \mathrm{eV}$ for transforming E1 to $\mathrm{E} 2$ and $0.28 \mathrm{eV}$ for transforming E2 to E1 [9].

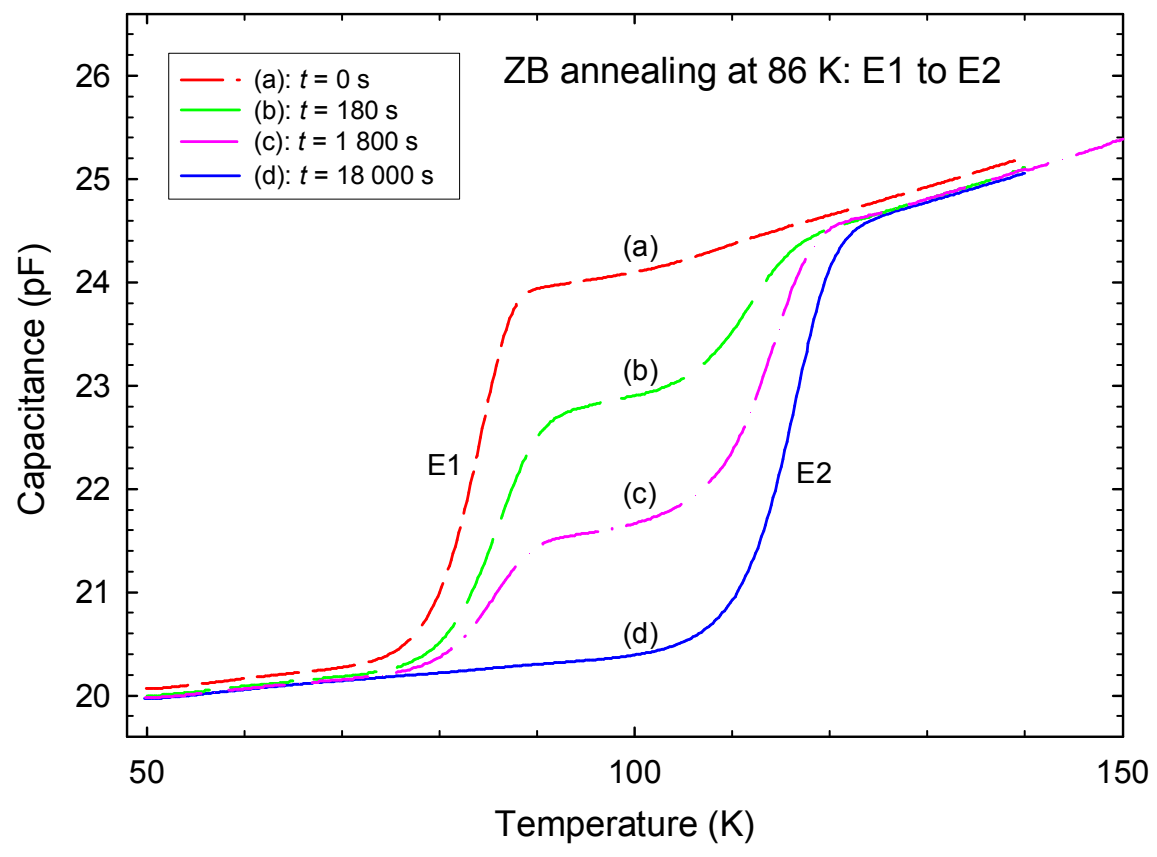

Fig. 3. C-T scans recorded up in temperature after annealing the sample at $86 \mathrm{~K}$ for different times as indicated in the figure. Annealing under ZB transforms E1 to E2.

\section{Conclusions}

The two most prominent defects introduced by $300 \mathrm{keV}$ Eu-ion implantation in HVPE grown $\mathrm{GaN}$ (followed by annealing at $1000{ }^{\circ} \mathrm{C}$ ) are the metastable defect pair E1 and E2 with activation enthalpies of $0.18 \mathrm{eV}$ and $0.27 \mathrm{eV}$, respectively. E1 and E2 can be reversibly removed during $\mathrm{ZB}$ and $\mathrm{RB}$ annealing cycles, respectively, and can be reversibly introduced during $\mathrm{RB}$ and $\mathrm{ZB}$ annealing cycles, respectively. Both these transformation processes follow first order kinetics. The origin of $\mathrm{E} 1$ and E2 are proposed to be related to implantation induced damage and not to Eu.

\section{Acknowledgements}

This work has been made possible by financial assistance from the South African National Research Foundation, the Fund for Scientific Research, Flanders (FWO), the Concerted Action of the KULeuven (GOA/2009/006), the Inter-university Attraction Pole (IAP P6/42) and the Center of Excellence Programme (INPAC EF/05/005). The Laplace DLTS software and hardware used here was received from L. Dobaczewski (Institute of Physics, Polish Academy of Science) and A. R Peaker (Centre for Electronic Materials Devices and Nanostructures, University of Manchester).

\section{References}

[1] S. Nakamura, G. Fasol, The Blue Laser Diode (Springer, Heidelberg, 1997).

[2] P.N. Favennec, H. L'Haridon, M. Salvi, D. Moutonnet, Y. Le Guillou, Electron. Lett. 25 (1989) 718.

[3] J.-S. Filhol, R. Jones, M.J. Shaw, P.R. Briddon, Appl. Phys. Lett. 84 (15) (2004) 2841.

[4] M. Mamor, V. Matias, and A. Vantomme, A. Colder, P. Marie, and P. Ruterana, Appl. Phys. Lett. 85, (2004) 2244.

[5] S.F. Song, W.D. Chen, C. Zhang, L. Bian, C.C. Hsu, L.W. Lu, Y.H. Zhang, J. Zhu, Appl. Phys. Lett. 86 (2005) 2111

[6] P. J. Janse van Rensburg, F.D. Auret, V. Matias and A. Vantomme, Physica B 404 (2009) 44114414 
[7] L. Dobaczewski, P. Kaczor, I.D. Hawkins, A.R. Peaker, J. Appl. Phys. 76 (1994) 194.

[8] G. D. Watkins and J. R. Troxell, Phys. Rev. Lett. 44 (1980) 593-596.

[9] To be submitted to Phys. Rev. B, September 2010 